Supporting Information for:

\title{
Using micro-Raman spectroscopy to investigate chemical composition, mixing states, and heterogeneous reactions of individual atmospheric particles
}

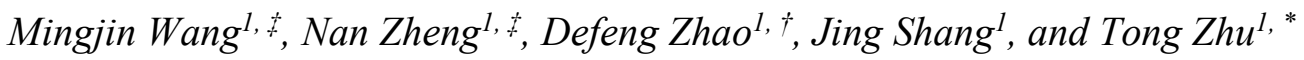

1 BIC-ESAT and SKL-ESPC, College of Environmental Sciences and Engineering, Peking University, Beijing, 100871, China.

$\dagger$ Present address: Dept. of Atmos. and Oceanic Sci. \& Inst. of Atmos. Sci., Fudan University, Shanghai, 200438, China.

$\$$ Mingjin Wang and Nan Zheng contributed equally to this work.

* Corresponding author. E-mail: tzhu@pku.edu.cn (Tong Zhu)

Number of pages: 10 (S1-S10)

Number of tables: 3 (Tables S1-S3)

Number of figures: 4 (Figures S1-S4) 


\section{Contents}

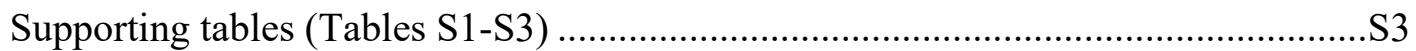

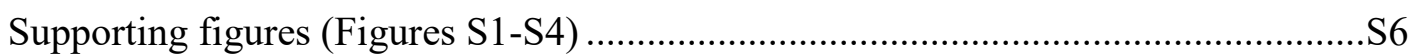

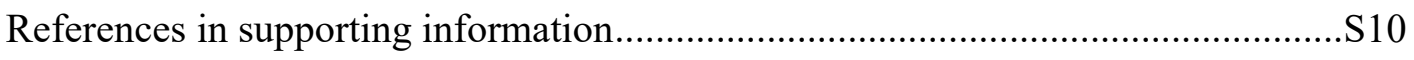




\section{Supporting tables}

Table S1. 24-hours average data of pollutant concentrations and meteorological parameters before sampling.

\begin{tabular}{llllllllll}
\hline $\begin{array}{l}\text { Sample } \\
\text { No. }^{a}\end{array}$ & $\begin{array}{l}\text { Samplin } \\
\text { g time }^{\mathrm{b}}\end{array}$ & $\begin{array}{l}\mathrm{NO}_{\mathrm{x}} \\
(\mathrm{ppb})\end{array}$ & $\begin{array}{l}\mathrm{SO}_{2} \\
(\mathrm{ppb})\end{array}$ & $\begin{array}{l}\mathrm{O}_{3} \\
(\mathrm{ppb})\end{array}$ & $\begin{array}{l}\mathrm{PM}_{2.5} \\
\left(\mu \mathrm{g} / \mathrm{m}^{3}\right)^{\mathrm{c}}\end{array}$ & $\begin{array}{l}\mathrm{T} \\
\left({ }^{\circ} \mathrm{C}\right)\end{array}$ & $\begin{array}{l}\mathrm{RH} \\
(\%)\end{array}$ & $\begin{array}{l}\mathrm{WS} \\
(\mathrm{m} / \mathrm{s})\end{array}$ & $\begin{array}{l}\mathrm{WD} \\
\left({ }^{\circ}\right)\end{array}$ \\
\hline $\mathrm{S} 0422$ & $17: 00: 00$ & 28.34 & 0.55 & 21.99 & 35 & 10.15 & 70.76 & 1.56 & 267.15 \\
$\mathrm{~S} 0423$ & $18: 00: 00$ & 60.29 & 1.61 & 17.88 & 20 & 13.63 & 40.04 & 1.56 & 253.84 \\
$\mathrm{~S} 0424$ & $15: 00: 00$ & 68.70 & 6.50 & 21.78 & 117 & 16.40 & 27.49 & 2.36 & 220.43 \\
$\mathrm{~S} 0425$ & $15: 00: 00$ & 31.69 & 8.09 & 38.36 & 135 & 17.20 & 51.01 & 3.12 & 203.74 \\
$\mathrm{~S} 0426$ & $15: 00: 00$ & 17.68 & 2.30 & 25.98 & 24 & 10.25 & 46.19 & 2.34 & 170.72 \\
$\mathrm{~S} 0429$ & $14: 00: 00$ & 8.10 & 0.50 & 31.76 & 19 & 12.86 & 24.12 & 4.29 & 179.17 \\
$\mathrm{~S} 0503$ & $14: 00: 00$ & 50.30 & 4.93 & 23.25 & 150 & 24.71 & 17.19 & 2.14 & 207.99 \\
$\mathrm{~S} 0504$ & $16: 00: 00$ & 44.23 & 9.59 & 31.31 & 136 & 21.79 & 43.41 & 1.79 & 155.62 \\
$\mathrm{~S} 0505$ & $10: 00: 00$ & 36.29 & 5.97 & 25.28 & 282 & 21.29 & 69.56 & 2.08 & 162.31 \\
$\mathrm{~S} 0506$ & $10: 00: 00$ & 11.38 & 0.74 & 37.05 & 16 & 19.30 & 33.99 & 4.76 & 176.68 \\
$\mathrm{~S} 0507$ & $16: 00: 00$ & 26.24 & 4.07 & 27.70 & 130 & 23.66 & 27.07 & 3.34 & 131.29 \\
\hline
\end{tabular}

Notes:

a: The sample No. is $\mathrm{S}$ (sample) $+\mathrm{xx}$ (month) $+\mathrm{xx}$ (date).

${ }^{\mathrm{b}}$ : Start time of the sampling. Local time (UTC/GMT+08:00, Beijing, China), 24-hour system.

c: Hourly $\mathrm{PM}_{2.5}$ of the sampling hour. The $\mathrm{PM}_{2.5}$ data are from the Mission China air quality monitoring program (U.S. Embassy Beijing Air Quality Monitor). Other data in this table are from Peking University station. 
Table S2. End time of sampling and Raman measurements for each sample and the intervals between them.

\begin{tabular}{llll}
\hline Sample No. & End time of sampling & End time of Raman measurements & Interval \\
\hline S0422 & $04 / 22 / 2010,17: 02: 00$ & $04 / 22 / 2010,19: 20: 43$ & 2 hours \\
S0423 & $04 / 23 / 2010,18: 02: 00$ & $04 / 23 / 2010,22: 23: 21$ & 4 hours \\
S0424 & $04 / 24 / 2010,15: 02: 00$ & $04 / 24 / 2010,17: 02: 11$ & 2 hours \\
S0425 & $04 / 25 / 2010,15: 02: 00$ & $04 / 25 / 2010,17: 10: 51$ & 2 hours \\
S0426 & $04 / 26 / 2010,15: 02: 00$ & $04 / 26 / 2010,17: 06: 24$ & 2 hours \\
S0429 & $04 / 29 / 2010,14: 02: 00$ & $05 / 07 / 2010,22: 01: 52$ & 8 days \\
S0503 & $05 / 03 / 2010,14: 02: 00$ & $05 / 09 / 2010,14: 50: 52$ & 6 days \\
S0504 & $05 / 04 / 2010,16: 02: 00$ & $05 / 10 / 2010,15: 45: 08$ & 6 days \\
S0505 & $05 / 05 / 2010,10: 02: 00$ & $05 / 11 / 2010,15: 45: 12$ & 6 days \\
S0506 & $05 / 06 / 2010,10: 02: 00$ & $05 / 13 / 2010,12: 24: 03$ & 7 days \\
S0507 & $05 / 07 / 2010,16: 02: 00$ & $05 / 14 / 2010,16: 05: 43$ & 7 days \\
\hline
\end{tabular}

Notes:

${ }^{a}$ : The sample No. is $\mathrm{S}$ (sample) $+\mathrm{xx}$ (month) $+\mathrm{xx}$ (date).

b: Local time (UTC/GMT+08:00, Beijing, China), 24-hour system. 
Table S3. Brief summary of four individual particle techniques. ${ }^{\mathrm{a}}$

\begin{tabular}{|c|c|c|c|c|}
\hline & Morphology & Chemical composition & Environment $\mathrm{t}^{\mathrm{b}}$ & Mode \\
\hline $\begin{array}{l}\text { SEM/TEM- } \\
\text { EDX }^{\mathrm{c}}\end{array}$ & $\begin{array}{l}\text { 2D particle shape, } \\
\text { SEM: } 3-6 \mathrm{~nm} \text { resolution, } \\
\text { TEM: } 0.1 \mathrm{~nm} \text { resolution }\end{array}$ & $\begin{array}{l}\text { Elemental composition, } \\
\text { Elements larger than } \\
\text { carbon }(\mathrm{C})\end{array}$ & $\begin{array}{l}\text { High vacuum, } \\
\text { volatile species } \\
\text { may be lost }\end{array}$ & Offline \\
\hline $\begin{array}{l}\text { Micro- } \\
\text { Raman } \\
\text { spectroscopy }\end{array}$ & $\begin{array}{l}\text { 2D particle shape, } \\
\text { optical images with } 200 \\
\text { nm resolution }\end{array}$ & $\begin{array}{l}\text { Molecules and } \\
\text { functional groups }\end{array}$ & $\begin{array}{l}\text { Normal } \\
\text { ambient } \\
\text { condition }\end{array}$ & Offline \\
\hline SERS $^{\mathrm{d}}$ & $\begin{array}{l}\text { 2D particle shape, } \\
\text { optical images with } 200 \\
\text { nm resolution }\end{array}$ & $\begin{array}{l}\text { Molecules and } \\
\text { functional groups, } \\
\text { enhanced signals, } \\
\text { nonlinear }\end{array}$ & $\begin{array}{l}\text { Normal } \\
\text { ambient } \\
\text { condition }\end{array}$ & Offline \\
\hline SPAMS $^{\mathrm{e}}$ & No information & $\begin{array}{l}\text { Ion spectra, fragment } \\
\text { ions }\end{array}$ & $\begin{array}{l}\text { High vacuum, } \\
\text { volatile species } \\
\text { may be lost }\end{array}$ & Online \\
\hline
\end{tabular}

Notes:

a: Part of the data in this table referred to Li et al. ${ }^{1}$

b: The environments in which individual particles were measured.

c: Scanning/transmission electron microscopy coupled with energy-dispersive X-ray spectroscopy.

d: Surface-enhanced Raman scattering.

e: Single-particle aerosol mass spectrometry. 


\section{Supporting figures}

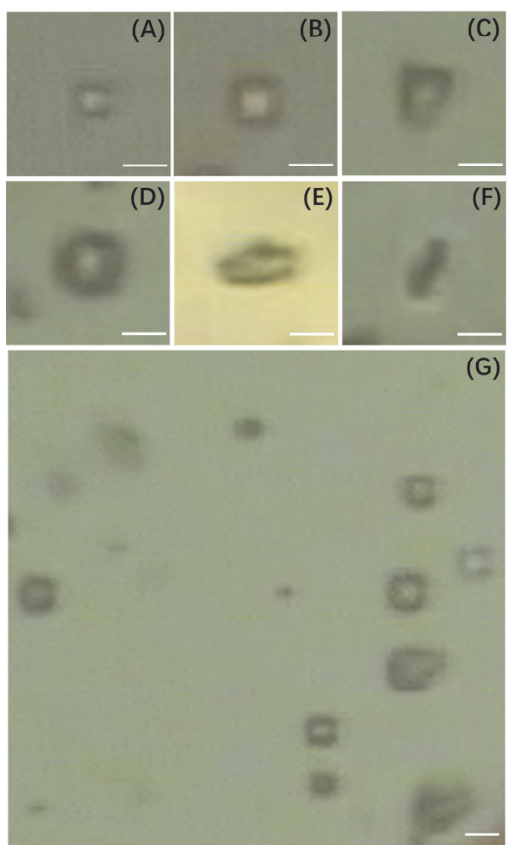

Figure S1. Optical images of (A) a $\left(\mathrm{NH}_{4}\right)_{2} \mathrm{SO}_{4}$ particle, (B) a $\mathrm{NH}_{4} \mathrm{NO}_{3}$ particle, (C) a $\mathrm{CaCO}_{3}$ particle, (D) a $\mathrm{Ca}\left(\mathrm{NO}_{3}\right)_{2}$ particle, $(\mathrm{E})$ an organic particle, $(\mathrm{F})$ a soot particle, and $(\mathrm{G})$ an area of S0426. The plotting scales in all images are $2 \mu \mathrm{m}$. 


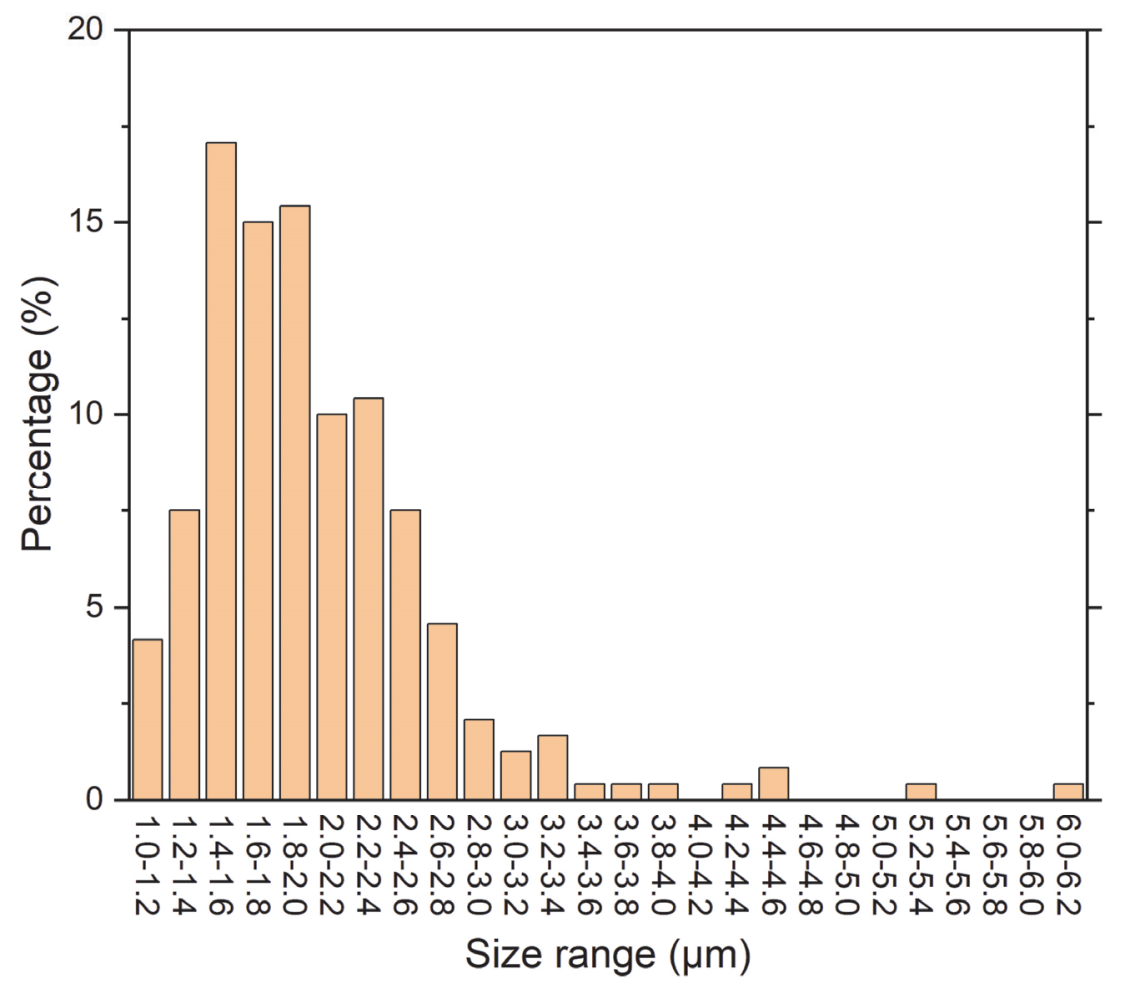

Figure S2. The number percentages of individual particles in each size range. 

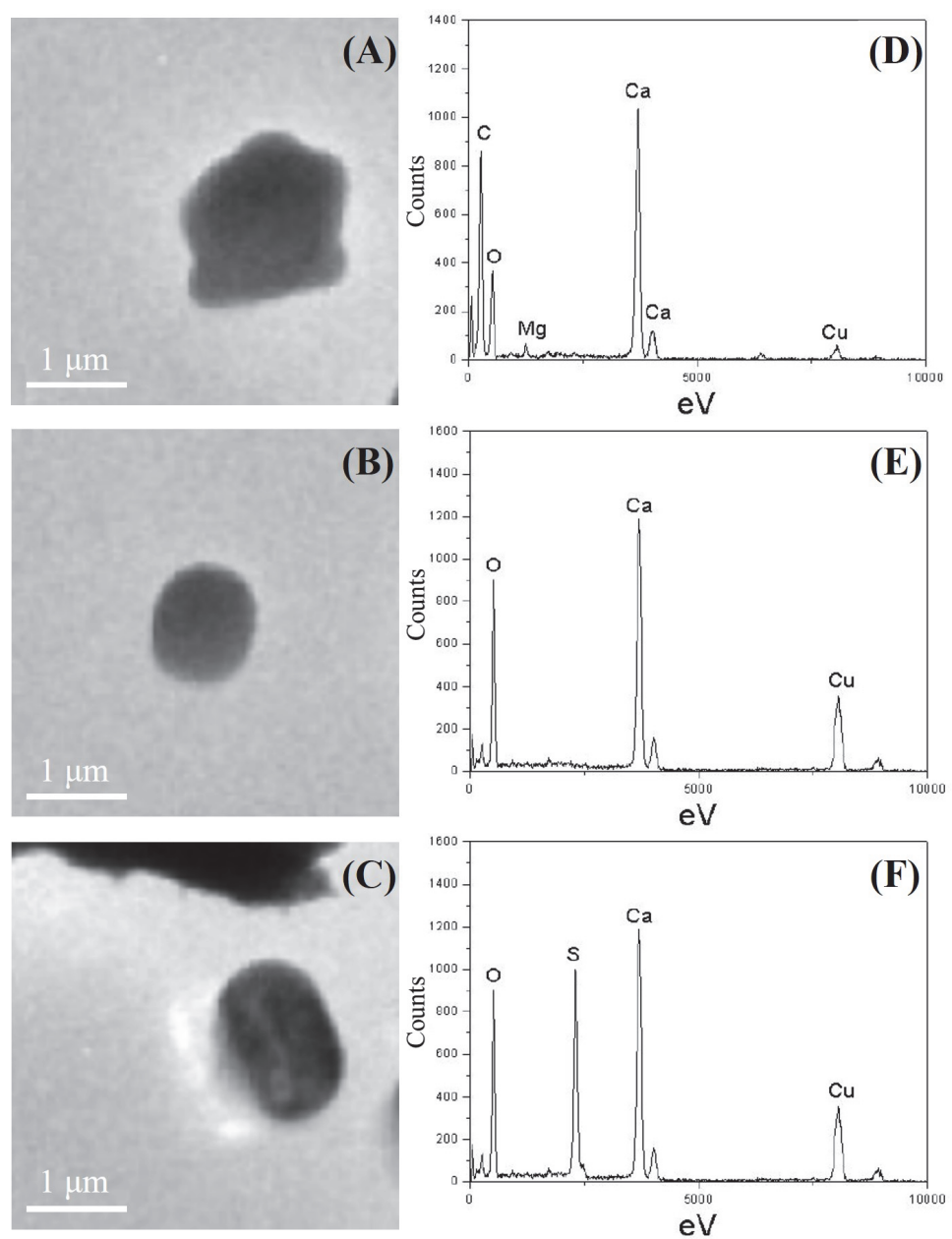

Figure S3. Left: TEM pictures of (A) a $\mathrm{CaCO}_{3}$ particle, (B) a $\mathrm{Ca}\left(\mathrm{NO}_{3}\right)_{2}$ particle, (C) a $\mathrm{CaSO}_{4}$ particle found in $\mathrm{S} 0506$. Right: EDX spectra of (D) the $\mathrm{CaCO}_{3}$ particle in (A), (E) the $\mathrm{Ca}\left(\mathrm{NO}_{3}\right)_{2}$ particle in $(\mathrm{B})$, and $(\mathrm{F})$ the $\mathrm{CaSO}_{4}$ particle in $(\mathrm{C})$. The signal of $\mathrm{N}$ in $\mathrm{EDX}$ spectrum is very low. ${ }^{2-4}$ In this study, no significant signal of $\mathrm{N}$ was detected in the EDX spectrum of the $\mathrm{Ca}\left(\mathrm{NO}_{3}\right)_{2}$ particle. So the $\mathrm{CaCO}_{3}$ and $\mathrm{CaSO}_{4}$ particles in (A) and (C) may contain $\mathrm{Ca}\left(\mathrm{NO}_{3}\right)_{2}$. The signal of $\mathrm{Cu}$ is from the copper grid. 

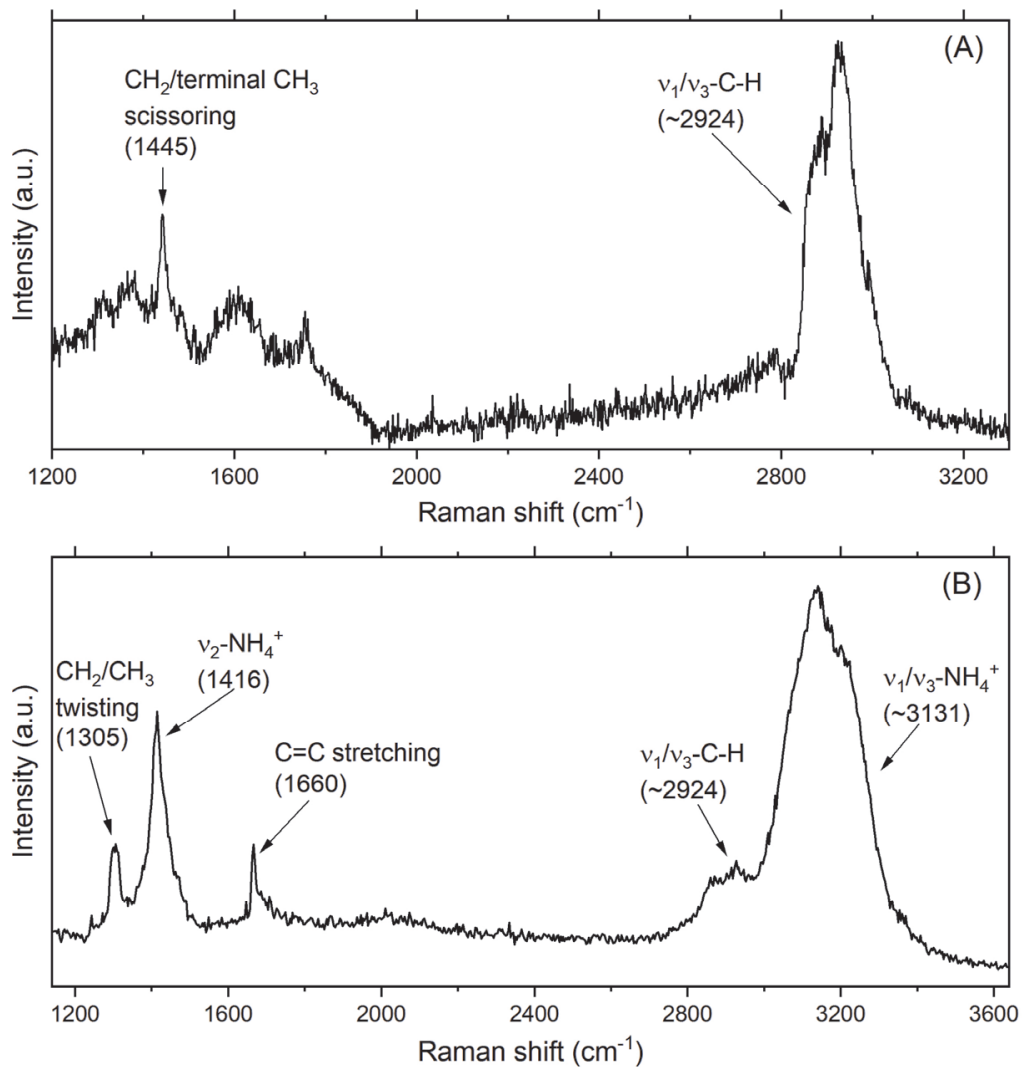

Figure S4. Raman peaks at (A) $1445 \mathrm{~cm}^{-1}\left(\mathrm{CH}_{2} /\right.$ terminal $\mathrm{CH}_{3}$ scissoring vibrations), (B) $1305 \mathrm{~cm}^{-1}\left(\mathrm{CH}_{2} / \mathrm{CH}_{3}\right.$ twisting vibrations $)$, and $1660 \mathrm{~cm}^{-1}(\mathrm{C}=\mathrm{C}$ stretching vibration $)$ observed in the Raman spectra of individual particles in this study. 


\section{References in supporting information}

(1) Li, W.; Shao, L.; Zhang, D.; Ro, C.-U.; Hu, M.; Bi, X.; Geng, H.; Matsuki, A.; Niu, H.; Chen, J. A review of single aerosol particle studies in the atmosphere of East Asia: morphology, mixing state, source, and heterogeneous reactions. J. Cleaner Prod. 2016, 112, 1330-1349.

(2) Hwang, H.; Ro, C.-U. Direct observation of nitrate and sulfate formations from mineral dust and sea-salts using low-Z particle electron probe X-ray microanalysis. Atmos. Environ. 2006, 40 (21), 3869-3880.

(3) Laskin, A.; Iedema, M. J.; Ichkovich, A.; Graber, E. R.; Taraniuk, I.; Rudich, Y. Direct observation of completely processed calcium carbonate dust particles. Faraday Discuss. 2005, 130, 453-468.

(4) Li, W. J.; Shao, L. Y. Observation of nitrate coatings on atmospheric mineral dust particles. Atmos. Chem. Phys. 2009, 9 (6), 1863-1871. 